Original Research Paper

\title{
On the Acclimatization of Pelagic Fish Species Juveniles in a Closed Environment: An Atlantic Horse Mackerel Case Study
}

\author{
${ }^{1,2}$ Charles Odilichukwu R. Okpala, ${ }^{1}$ Giacomo Sardo and ${ }^{1}$ Sergio Vitale \\ ${ }^{1}$ Istituto per l'Ambiente Marino Costiero-Consiglio Nazionale delle Ricerche (IAMC-CNR), \\ 91026 Mazara del Vallo-TP, Italy \\ ${ }^{2}$ Educare and Skills Training Network, Middlesex HA8 8EJ, United Kingdom
}

Article history

Received: 02-01-2017

Revised: 01-04-2017

Accepted: 13-04-2017

Corresponding Author:

Charles Odilichukwu R. Okpala

1. Istituto Per l'Ambiente

Marino Costiero - Consiglio

Nazionale delle Ricerche

(IAMC-CNR), 91026 Mazara

del Vallo-TP, Italy

2. Educare and Skills Training

Network, Middlesex HA8 8EJ,

United Kingdom

Email: charlesokpala@gmail.com

\begin{abstract}
Understanding how fish species acclimatize to a new environment may help to improve aquatic/fisheries resource management. Further, baseline information specific to behavior patterns of (pelagic) fish species juveniles particularly during acclimatization appears unreported to best of our knowledge. Therefore, the current study was goaled to examine the acclimatization of pelagic fish species juveniles in a closed environment using Trachurus trachurus as a case reference. Eighteen Trachurus fish juveniles were maintained in well-kept seawater-filled aquarium under laboratory/room conditions of controlled temperature. The characteristic observations of fish aggregation, fish-to-feed movement and fish-feed selection that varied across begin (days 1-5), middle (days 6-15) and end (days 16-20) phases provided substantiations about either how and when Trachurus spp. juveniles started, increased and or achieved complete/full acclimatization behavior. The initial peak (laboratory $/$ room $=\sim 18^{\circ} \mathrm{C}$; water $=\sim 20^{\circ} \mathrm{C}$ ) temperatures of 20-day acclimatization period would stabilize with time. Along with the abovementioned characteristic observations, water temperatures seemed to fluctuate more compared to laboratory/room. Overall, the current study provides noteworthy baseline knowledge about pelagic fish juveniles' behavior(s)/pattern(s) in closed environment and useful reference for future acclimatization studies.
\end{abstract}

Keywords: Behavior Pattern, Trachurus trachurus, Fish-to-Fish Movement, Fish-Feed Selection, Fish Aggregation, Temperature

\section{Introduction}

Atlantic horse mackerel (Trachurus trachurus) is among well-known economically important pelagic fish species reported to inhabit (fresh) marine sites such as Adriatic, Atlantic northeastern, Black and Mediterranean Seas. Although it is considered among demersal fisheries within Mediterranean Basin (Šantić et al., 2005), it has for three decades or more been known as 'stickleback' in Canada (http://solmarfoods.com/horse-mackeral/: Accessed 04/09/2016). Trachurus spp. has been positioned within multiple specie models of North Sea fish stock assessment due to its increasing population. Also, the large stock biomass of Trachurus spp. represents the latter as considerable predators of other fish species (Herrmann and Enders, 2000). The stomach contents of Trachurus spp. have revealed both crustacea (Euphausiacea, Mysidacea, Decapoda) and fish larvae (Konstantinos and Karponzi, 2002). The feeding intensity of Trachurus spp. remains diverse and varied (Bowman and Bowman, 1980). Besides, the feeding data about Trachurus spp. already generated at Mediterranean Basin has been largely based on (feed/food) composition compared to (feeding) intensity (Jardas et al., 2004). In addition, the management of Trachurus spp. in Northeast Atlantic as well as those coordinated by International Council for Exploration of the Sea (ICES) has come with challenges probably due to assessment uncertainties (Ndjaula et al., 2009).

At natural environment/habitat, the movement of fish species is largely without any limitation. But this is not so when fish species are subject to a closed environment. In this context, a period of acclimatization has always been deemed needful as routinely observed at many fishrelated studies (Hunter, 1968; Marchesan et al., 2005; 2009). Despite exposure to new environment to induce some stress to fish, the environment in which fish species are found also contributes to either regulate and 
or set boundaries on (its) physiological performance (Schreck, 2000). Notably, shoaling-a common acclimatization/adaptation behavior has been reported among studies involving a number of fish species (Pavlov and Kasumyan, 2000). Consequently, how fish species form groups can be characterized by (high) within-and between-individual variations that may well depend on individual species size, feed availability and predation risks (Seppälaä et al., 2008). Regardless of the intended (research) objectives, majority of previous studies that placed fish species within closed environment have been widely understood to use juveniles obviously due to the small size, which makes the conduct of such studies feasible and plausible in laboratory-contained aquarium(s). To unravel more information about this aspect of fishery sciences will require the formulation of additional research questions, which according to Okpala (2017) has been considered beneficial for the definition, collection and reporting of relevant information. Despite substantial scientific literature currently available about pelagic fish species and to the best of authors' knowledge, baseline information specific to behavior patterns of (pelagic) fish species juveniles particularly during acclimatization appears not reported. To our knowledge, this is the first report to establish the acclimatization of pelagic fish species juveniles in a closed environment using Trachurus trachurus as a case model/reference. Combining visual evidence by and descriptive case approach, the acclimatization behavior(s)/pattern (s) has been qualified.

\section{Materials and Methods}

\section{Ethical Considerations and Collection of Samples}

Before this study, the ethical approval was sought for at IAMC-CNR, which upon approval, the experimental study was performed.

At end of a spawning season following that reported by Murta (2000), eighteen (18) Trachurus spp. juvenile fish samples for this study were caught off the coast of Mazara del Vallo (Italy) across Latitude $37^{\circ} 34^{\prime}$ 40''/Longitude 12 $34^{\prime}$ '01' ' (Depth $45 \mathrm{~m}$ ) up to Latitude $37^{\circ} 33^{\prime} 13^{\prime}$ '/Longitude $12^{\circ} 34^{\prime} 86^{\prime}$ ' (Depth $50 \mathrm{~m}$ ), within the confines of International bottom trawl survey in Mediterranean (MEDITS): An European Union (EU) project targeting repetitive trawl vessel survey that assess demersal resources after fishing activities operating at depth range of 10-800 m using low mesh sized nets with codend (10 mm) (MEDITS, 2012). After catch and using an aerated container, the Trachurus spp. fish samples have been transferred to laboratory of IAMC-CNR within same (catch day) morning.

\section{Aquarium (Tank + Water + Samples) Maintenance}

Seawater obtained from the rocky shores coasts of Mazara del Vallo have been used to fill a rectangular
(Length $\times$ Width $\times$ Height: $1.51 \times 0.51 \times 0.66 \mathrm{~m})$ glass tank of maximum $400 \mathrm{~L}$ volume capacity, equipped with submersible Bioflow Filter (Juwel ${ }^{\circledR}$ Aquarium $\mathrm{GmbH}$, Rotenburg, Deutschland) and internal Blueskimmer 550 (Ferplast S.p.A., Castelgomberto, Italy) respectively purposed to remove organic particles/other substances in suspension and avoid the accumulation of toxic chemicals that may emanate from fish excrete/feed remnants-all geared towards quality aquarium/tank maintenance. In addition, a supercharger was supplemented for aquarium water oxygenation. In this specific aquarium facility and throughout the studied period, the Trachurus spp. samples have been maintained. To ensure consistent/continued quality maintenance of aquarium water, in addition to the cleaning of filters and approximately $20 \%$ of current water replacement with freshly obtained seawater via help of water pump, there was simultaneous clean-up of tank bottom to rid-off inedible/undesirable (waste) particles/remnants.

\section{Feeding of Samples}

Since stomach analysis of Trachurus spp. previously reported has shown crustacea as predominant diet constituent (Jardas et al., 2004), Trachurus spp. juveniles of this study were fed with shrimp freshly harvested thereafter freeze stored $\left(\sim-18^{\circ} \mathrm{C}\right)$. To prepare the fish feed, frozen shrimp were thawed followed by manual maceration (little pieces not exceeding $0.5 \mathrm{~cm}$ ) in view to enhance its (Trachurus spp.) feeding intensity. Further, driblets of macerated full-dry Artemia shrimp (Sera $^{\mathrm{TM}}$, D52518 Heinsberg, Germany) and Gammarus shrimp (Family Pet S.r.L., Toscanella di Dozza, Italy) were initially administered alone but subsequently supplemented on feed menu in the view to fortify the entire feed diet. Given that high feeding intensity of Trachurus spp. has been reported at night and during early mornings (Jardas et al., 2004), feed to samples ad libitum (of this study) were administered at scheduled times per day on different areas of evenly on water surface to allow for equal feed distribution/reception.

\section{Study Design and Data Collection}

Specifically, this current study was designed to establish the acclimatization of pelagic fish species juveniles within a closed environment in view to understand behavior pattern using Trachurus spp. as a case reference. Using a descriptive approach and based on eighteen fish samples, all (emergent) acclimatization attributes were documented based on direct visual evidence(s) (daily) of observed behavior(s)/pattern(s). In order to qualify as well as quantify all documented acclimatization attributes to arrive at descriptive remarks, series of focus group discussions were performed on all documented visual evidences so as to identify/tease-out the various connects between observed 
fish behavior(s)/pattern(s) and its corresponding attributes. This has allowed for subsequent discussions about trends of observed acclimatization attributes with time. Since the studied fish species required temperature environment that likens to that of the natural situation, the temperatures of both water and laboratory/room were considered alongside acclimatization attributes of fish aggregation, fish-to-feed movement and fish-feed selection. Temperatures of laboratory/room was controlled by airconditioning (Split unit Ariagel ${ }^{\mathrm{TM}}$, APW 325, of Monclick S.r.L., Monza e Brianza, Italy) and assayed by electronic thermometer (Diligence EV N2014, Comark Instruments, Norwich NR6 6JB, United Kingdom), the latter also used to determine the water temperature. Essentially, this study period lasted for 20 days under natural photoperiod of either twelve hours of light (08.00-20.00 h) or dark (20.00-08.00 h) conditions.

\section{Quantification of Observed Data}

The monitoring of temperature data was performed before feeding at mornings (8-9 am), afternoons (1-2 pm) and evenings (6-7 pm). At these same times, observations of characteristic behaviors were performed close to feeding and during feeding times in the view to communicate the events of fish-feed selection (how fish were able to select their administered feed $\{s\}$ ), fish-to-feed movement (how fish moved during the administration and reception of feed $\{s\})$ as well as fish aggregation. To compare laboratory/room and water temperatures, the analysis of variance (ANOVA) were carried out using Tukey's simultaneous test at $95 \%$ confidence interval.

\section{Results and Discussion}

The current study lasted for 20 days, the latter divided into three major phases, i.e., 'begin' (days 1-5), 'middle' (days 6-15) and 'end' (days 16-20). In addition, the middle phase (further) has been sub-divided into 'first' (days 6-10) and 'second' (days 11-15) aspects. As schematically represented in Fig. 1, the acclimatization attributes of Trachurus spp. juveniles in closed environment appeared to largely vary between the abovementioned phases.

At the 'begin' phase (days 1-5), there was low/minimal fish-feed selection as fish species persisted to refuse/reject/spit-out the administered feed even after different/repeated feeding attempts. Change of habitat from open/spaced sea to a closed/limited environment could be a cause, which might probably had resulted in some disorientation/stress to explain (their) regular shoaling and to suggest increased protective instincts. Both regular shoaling and feed rejection might probably link to indistinct/unclear fish-feed hierarchy (Fig. 1). Regardless of size, the Trachurus spp. does show collective/synchronized motion, which appears to follow a coordinated manner (Herbert-Read et al., 2011). Herein, between days 3-5 (late part of begin phase), some fishfeed hierarchy seemed introduced as fish species began to accept/approve alternative feed concurrent to moderate shoaling (Fig. 1). Such result can serve as clue(s) that Trachurus spp. fish began to acclimatize to the new (closed) environment. Clearly, the positive association between fish-feed selection and fish-to-feed movement, but negative association between fish-feed selection and fish aggregation characterized this begin phase.

At the 'middle' phase (days 6-15), the Trachurus spp. species at the 'first' part (days 6-10) would continue to accept/approve the administered/alternative feed, which made the complete/total fish-feed selection to specific feed (types) look more apparent (Fig. 1). This observation can suggest the fish species possess the capacity to differentiate between (administered) artificial and natural feed types. Further, 'first' aspect of middle phase also revealed initially distinct/evident feed-fish hierarchy dropping to moderate with much less shoaling (fish aggregation), which may point to some increased acclimatization efforts by the juvenile fish samples. The progress in acclimatization (specific to the 'first' aspect of middle phase) can corroborate with somewhat less feeding hierarchy of Trachurus spp. fish, which may well suggest less protective instincts. Notably, the positive association between fish aggregation and fish-to-feed movement, negative association between fish-feed selection against both fish-tofeed movement and fish aggregation characterized this 'first' aspect of middle phase (Fig. 1). At the 'second' aspect of middle phase (days 11-15), a complete/total fishfeed selection seemed conclusive as the administered feed got rapidly accepted. Compared to days 6-10, fish aggregation amid initial decreases looked stable between days 11-15 but rather concomitant with the apparently less fish-to-feed movement and fish-feed hierarchy. Between days 11-15 also, the smaller fish during the feeding periods remained below bigger ones near bottom of water column. Collectively, the rather decreasing but stable trend of both fish-to-feed movement and fish aggregation in contrast to continuously stable fish-feed selection characterized this 'second' aspects of middle phase.

At the 'end' phase (days 16-20) of this study, the characteristics of fish-feed selection, fish-to-feed movement and fish aggregation of Trachurus spp. fish species appeared consistent, which seemed to indicate that complete/full acclimatization had been reached (Fig. 1). Specific to this 'end' phase, the fish continuously associated the feeder (operator) with feed demonstrated by left/right movements in water column following feeding tray held outside the aquarium. Since the studied stomach contents of horse mackerel have revealed a wide range of (smaller) fish types (Konstantinos and Karponzi, 2002), juvenile (pelagic) fish species upon complete/full acclimatization at closed environment may well possess some (increasing) feeding capacity. We therefore believe that the degree/extent of space of closed (new) environment can directly reflect how fish species eventually acclimatize. Thus, the limitedness of space within a given water body would strongly influence the acclimatization period of juvenile fish species. 


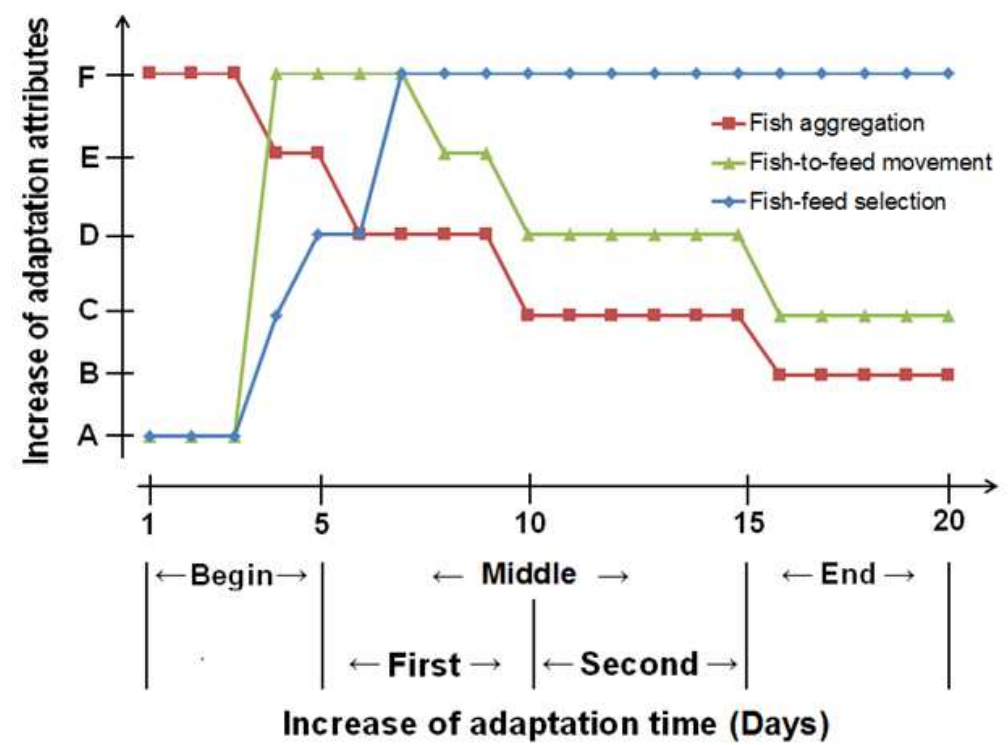

Fig. 1. Schematic representation of changes in acclimatization attributes of Trachurus trachurus juveniles with time in closed environment. Acclimatization attributes include: 'A' refers to either absent or attempt (where 'attempt' applies only to 'fishfeed selection'); 'B' refers to little (applies to all attributes); 'C' refers to either less or approval (where 'approval' applies only to 'fish-feed selection'); 'D' refers to either moderate or acceptance (where 'acceptance' applies only to 'fish-feed selection'); 'E' refers to less regular (applies to all attributes); ' $F$ ' refers to either high or total or distinct or regular (where 'total' applies only to fish-feed selection; 'distinct' applies only to 'fish-to-feed movement'; and 'regular' applies only to 'fish aggregation')

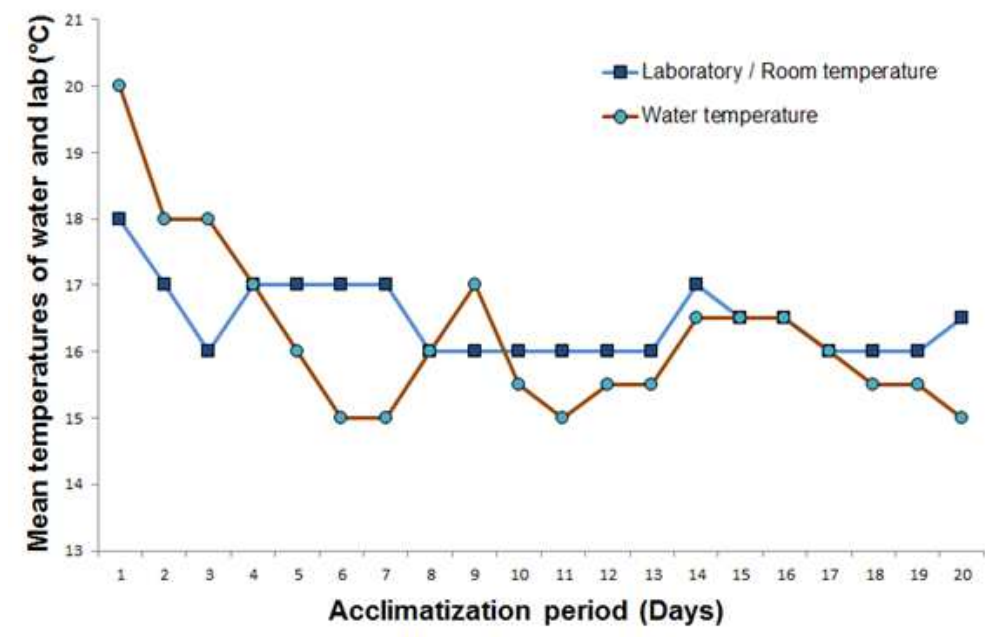

Fig. 2. Changes in mean water and laboratory/room temperatures during the acclimatization periods of Trachurus trachurus juveniles in a closed environment

As was observed in this current study, differences in fish size seemed more apparent in the progress of acclimatization time. Given that temperature is a prerequisite parameter and both room and water temperatures had to be determined/measured, the studied fish species had to be kept at the temperature environment that likened to natural situation. Under aquarium conditions therefore slight differences are well known to occur between room and water temperatures where fish species are kept/ maintained. And such temperature differences remain among management strategies applicable to any given aquatic ecosystem (Boubeé et al., 1997). The mean laboratory/room and water temperatures of current study would vary during the acclimatization of Trachurus spp. juveniles. Specifically, the initial peak (laboratory $/$ room $=\sim 18^{\circ} \mathrm{C}$; water $=\sim 20^{\circ} \mathrm{C}$ ) temperatures can be seen to stabilize subsequently. Moreover, the water temperatures seemed to fluctuate more compared with those of laboratory/room. Plausibly, the laboratory/room temperature might be moderating the water (Fig. 2). 


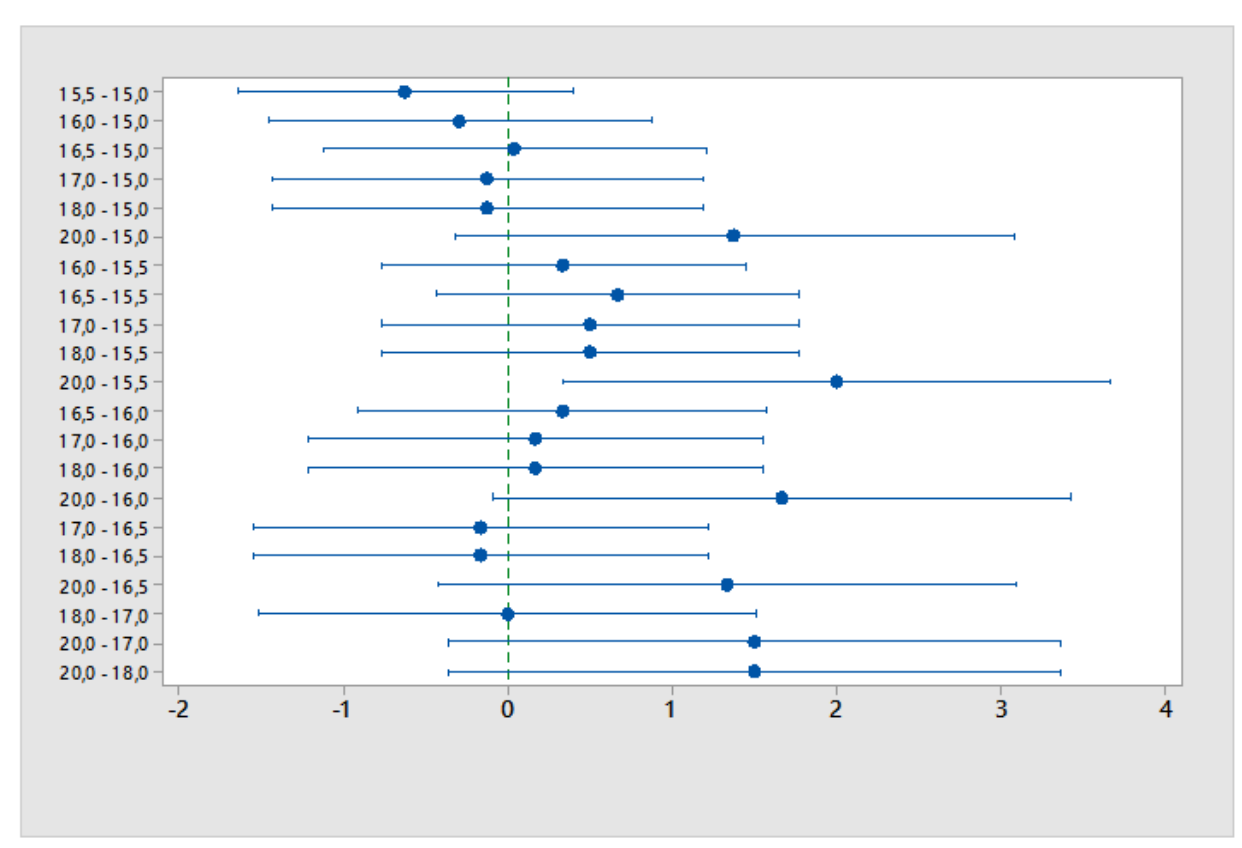

Fig. 3. Tukey's pairwise comparisons of temperatures (laboratory/room Vs water) at $95 \%$ confidence

Table 1. Summary of (emergent) fish acclimatization behavior(s)/pattern(s) applicable to Trachurus trachurus juveniles of this study

\begin{tabular}{|c|c|c|c|}
\hline \multicolumn{2}{|l|}{ Behaviors } & \multicolumn{2}{|l|}{ Patterns } \\
\hline Attributes & Remarks & Attributes & Remarks \\
\hline Feeding selection & $\begin{array}{l}\text { Movement towards feed } \\
\text { type }>\text { Feed bite }>\text { Feed rejection }> \\
\text { Movement towards }>\text { Another feed type }> \\
\text { Feed bite }>\text { No feed rejection }\end{array}$ & Fish-feed selection & $\begin{array}{l}\text { Complete/total feed selection } \\
\text { Acceptance feed selection } \\
\text { Approval feed selection } \\
\text { Attempt feed selection }\end{array}$ \\
\hline Feed acceptance & $\begin{array}{l}\text { Movement towards the feed } \\
>\text { Feed bite }>\text { No feed rejection }> \\
\text { Feed linkage with the feeder }\end{array}$ & Fish aggregation & $\begin{array}{l}\text { Regular shoaling } \\
\text { Moderate shoaling } \\
\text { Less moderate shoaling } \\
\text { Little/minimal shoaling }\end{array}$ \\
\hline Feed-Fish hierarchy & $\begin{array}{l}\text { Small fish stay at bottom/ } \\
\text { no upward attempt(s) } \\
\text { Feeding on remnants from bigger fish } \\
\text { Small fish stay below the bigger ones } \\
\text { (water column) }\end{array}$ & Fish-to-feed movement & $\begin{array}{l}\text { Distinct/evident feeding hierarchy } \\
\text { Less distinct feeding hierarchy } \\
\text { Moderate feeding hierarchy } \\
\text { Little feeding hierarchy } \\
\text { Absent feeding hierarchy }\end{array}$ \\
\hline
\end{tabular}

Further, both laboratory/room and water temperatures respectively showed $16.50 \pm 0.13$ and $16.26 \pm 0.30^{\circ} \mathrm{C}$ (mean \pm standard error \{of the mean\}). Even though these (abovementioned) temperatures seemed relatively close, Tukey's pairwise comparisons test (Fig. 3) showed them to be statistically different $(\mathrm{P}=0.037 ; \mathrm{F}=3.19$; $\mathrm{R}$-sq (adj. $)=$ $40.9 \%$ ). Besides, temperature contributes to control factors that affect fish internal medium to influence activities of metabolites (Schreck, 2000). Comparing Fig. 1 and 2 also some association between the stability of water temperature and attributes of fish-feed selection, fish-to-feed movement and fish aggregation seems plausible visually and this requires further clarifications/investigations (at the future studies) to help ascertain this proposition. A closer look at Fig. 2, however, both laboratory/room and water temperatures during acclimatization period would overlap but a few times. All the emergent behavior(s)/pattern(s) describing abovementioned acclimatization attributes of Trachurus spp. of this study is summarized in Table 1. The remarks of acclimatization behavior of feeding selection and feed acceptance were indicated with directions, but not so for fish-feed hierarchy. Hereafter, readers of this current research communication can appreciate how the observed step-wise acclimatization behavior(s) can translate into patterns of fish-feed selection, fish aggregation and fish-to-feed movement.

\section{Concluding Remarks}

For the first time, the acclimatization of pelagic fish species juveniles within closed environment using Trachurus spp. as a case reference was studied. Whereas some readers may perceive this study as chiefly descriptive, the information provided is noteworthy and goes beyond 
feeding behavior of such juvenile Trachurus fish species. Not only supplementing the existing body of knowledge, this current study provides improved understanding about acclimatization of fish juvenile in closed environment. Importantly, it equally serves as baseline reference useful for future investigations about other related (pelagic and non-pelagic) economic important fish species. Also, future studies involving such species should focus on the degree of applicability and consistency of behavior patterns as reported in current study, as generated data may well help to provide new information that would deepen the understanding about how temperature (laboratory/room and water) actually associates with fish-feed selection, fish-to-feed movement and fish aggregation.

\section{Acknowledgement}

Thanks to MEDITS for the funding support for this study. Thanks to Dr. Fabio Fiorentino and Dotts. Gioacchino Bono, Fabio Falsone, Danilo Scannella, Michele Geraci and Virginia Carrozzi for logistical support.

\section{Author's Contributions}

Charles Odilichukwu R. Okpala: Participated to initiate, conceptualize and design of study, rendered support to experimental work and data analysis and prepared the initial manuscript draft up to final stages.

Giacomo Sardo: Participated to initiate, conceptualize and design of study, performed the experiment/lab work, performed the data analyses and prepared the initial manuscript draft up to final stages.

Sergio Vitale: Participated to initiate, conceptualize and design of study, rendered support to experimental work, rendered support to data analysis and contributed to fine tune the manuscript up to the final draft.

\section{Competing Interests}

All the authors approve the manuscript and declare that there is no conflict of interest regarding the publication of this paper.

\section{References}

Boubeé, J.A.T., L.D. Tracie, D.W. West and R.F.G. Barrier, 1997. Avoidance of suspended sediment by the juvenile migratory stage of six New Zealand native fish species. New Zealand J. Mar. Freshwater Res., 31: 61-69. DOI: 10.1080/00288330.1997.9516745

Bowman, R.E. and E.W. Bowman, 1980. Diurnal variation in the feeding intensity and catchability of silver hake (Merluccius bilinearis). Can. J. Fish. Aqua. Sci., 37: 1565-1572. DOI: 10.1139/f80-202

Herbert-Read, J.E., A. Perna, R.P. Mann, T.M. Schaerf and D.J.T. Sumpter et al., 2011. Inferring the rules of interaction of shoaling fish. Proc. Nat. Acad. Sci., 108: 18726-18731. DOI: 10.1073/pnas. 1109355108
Herrmann, J.P. and E.C. Enders, 2000. Effect of body size on the standard metabolism of horse mackerel. J. Fish. Biol., 57: 746-760. DOI: $10.1111 / \mathrm{j} .10958649 .2000$. tb00272.x

Hunter, J.R., 1968. Effects of light on schooling and feeding of jack mackerel, Trachurus symmetricus. J. Fish. Board Can., 25: 393-407. DOI: 10.1139/f68-031

Jardas, I., Santic' M. and A. Pallaoro, 2004. Diet composition and feeding intensity of horse mackerel, Trachurus trachurus (Osteichthyes: Carangidae) in the eastern Adriatic. Mar. Biology, 144: 1051-1056. DOI 10.1007/s00227-003-1281-7

Konstantinos, I.S. and V.S. Karponzi, 2002. Feeding habits and trophic levels of Mediterranean fish. Rev. Fish Biology Fisheries, 11: 217-254. DOI: $10.1023 / \mathrm{A}: 1020556722822$.

Marchesan, M., M. Spoto, L. Verginella and E.A. Ferrero, 2005. Behavioural effects of artificial light on fish species of commercial interest. Fish. Res., 73: 171-185. DOI: $10.1016 /$ j.fishres.2004.12.009

Marchesan, M., M. Spoto and E.A. Ferrero, 2009. Impact of artificial light on behavioural patterns of coastal fishes of conservation interest. Varstvo Narave, 22: 117-136.

MEDITS, 2012. MEDITS-Handbook. Revision n. 6, April 2012, MEDITS Working Group, pp: 92.

Murta, A.G., 2000. Morphological variation of horse mackerel (Trachurus trachurus) in the Iberian and North African Atlantic: Implications for stock identification. ICES J. Mar. Sci., 57: 1240-1248. DOI: $10.1006 /$ jmsc. 2000.0810

Ndjaula, H.O.N., T. Hansen, M. Krüger-Johnsen and O.S. Kjeibu, 2009. Oocyte development in captive Atlantic horse mackerel Trachurus trachurus. ICES J. Mar. Sci., 66: 623-630. DOI: 10.1093/icesjms/fsp032

Okpala, C.O.R., 2017. Reflecting in the woods: Can it help to enhance the formulation of (our) research questions? BioResources J. Online (In-press).

Pavlov, D.S. and A.O. Kasumyan, 2000. Patterns and mechanisms of schooling behavior in fish: A review. J. Ichthyol., 40: S163-S231.

Šantić, M., I. Jardas and A. Pallaoro, 2005. Feeding habits of horse mackerel, Trachurus trachurus (Linneaus, 1758), from the central Adriatic Sea. J. Applied Ichthyol., 5: 125-130. DOI: $10.1111 / \mathrm{j} .1439-0426.2004 .00603 . \mathrm{x}$

Schreck, C.B., 2000. Accumulation and Long Term Effects of Stress in Fish. In: The Biology of Animal Stress: Basic Principles and Implications for Animal Welfare, Moberg, G.P. and J.A. Mench (Eds.), CABI, Wallingford, ISBN-10: 0851999301, pp: 377.

Seppälaä, O., A. Karvonen and T. Valtonen, 2008. Shoaling behaviour of fish under parasitism and predation risk. Animal Behav., 75: 145-150. DOI: 10.1016/j.anbehav.2007.04.022 